\title{
Serum long-chain n-3 polyunsaturated fatty acids, methylmercury and blood pressure in an older population
}

\author{
Jyrki K Virtanen ${ }^{1}$, Asenath N Nyantika ${ }^{1,2}$, Jussi Kauhanen ${ }^{1}$, Sari Voutilainen ${ }^{1}$ and Tomi-Pekka Tuomainen ${ }^{1}$
}

Fish or fish oil consumption has been associated with lower blood pressure. Fish may also contain methylmercury, which has been associated with cardiovascular diseases and higher blood pressure. Our aim was to study the associations of serum long-chain n-3 polyunsaturated fatty acids (PUFA) eicosapentaenoic acid (EPA), docosapentaenoic acid (DPA) and docosahexaenoic acid (DHA), mainly reflecting fish or fish oil intake, and hair mercury concentration with blood pressure. Data were available for 848 men and 909 women from the Kuopio Ischaemic Heart Disease Risk Factor Study, aged 53-73 years. We excluded participants with ischemic heart disease, stroke, diabetes or hypertension treatment, leaving 396 men and 372 women. Log-transformed values were used to study the associations. The mean serum concentrations were $1.63 \%$ (s.d. 0.91 ) for EPA, $0.77 \%$ (s.d. 0.16 ) for DPA and $2.73 \%$ (s.d. 0.90 ) for DHA of all serum fatty acids. Multivariate-adjusted serum EPA + DPA + DHA was associated with lower systolic blood pressure $(\beta=-4.50,95 \%$ confidence interval $(\mathrm{Cl})-8.02--0.99)$ and pulse pressure $(\beta=-4.41,95 \% \mathrm{Cl}-6.95--1.87)$, but not with diastolic blood pressure $(\beta=-0.45,95 \% \mathrm{Cl}-2.31-1.52)$. The associations were similar with EPA, DPA and DHA evaluated individually. The mean hair mercury concentration was $1.42 \mu \mathrm{g} \mathrm{g}^{-1}$ (s.d. 1.54). Hair mercury was not associated with blood pressure and it did not modify the association between PUFA and blood pressure. These results suggest that higher serum long-chain n-3 PUFA concentration has a modest inverse association with blood pressure in older men and women.

Hypertension Research (2012) 35, 1000-1004; doi:10.1038/hr.2012.80; published online 7 June 2012

Keywords: blood pressure; cross-sectional study; fatty acids; fish; mercury

\section{INTRODUCTION}

Hypertension continues to be a common cardiovascular disorder and it is a major risk factor for cardiovascular disease (CVD). Fish and fish oil consumption has been associated with reduced risk of CVD, for example sudden death and ischemic stroke. ${ }^{1}$ In population-based observational studies, higher consumption of fish or long-chain n-3 polyunsaturated fatty acids (PUFA) from fish have been associated with lower blood pressure in the general population ${ }^{2-5}$ and also in healthy, non-hypertensive participants. ${ }^{5}$ In meta-analyses of randomized controlled trials (RCT), fish oil supplementation has been shown to lower blood pressure, and the effect has been stronger in hypertensive subjects than in normotensive subjects. ${ }^{6-8}$ Intervention studies have also investigated the effect of increased fish consumption on blood pressure reduction, with diverse results. ${ }^{9-13}$ The possible mechanisms that may explain the inverse association between serum long-chain n-3 PUFA and blood pressure include improved vasodilation and vasoreactivity ${ }^{14,15}$ and arterial compliance, ${ }^{16}$ and reduced heart rate ${ }^{17}$ and vascular wall thickness. ${ }^{18}$
Fish is also a major source of methylmercury, an environmental contaminant. Previously, methylmercury exposure was associated with increased risk of CVD and with attenuation of the beneficial impact of long-chain n-3 PUFA on CVD risk in our Kuopio Ischaemic Heart Disease Risk Factor (KIHD) Study. ${ }^{19}$ Mercury has also been associated with blood pressure in other, but mainly highly exposed study populations. ${ }^{20-23}$ However, little is known about the relationship in populations with low to moderate exposure levels.

Furthermore, there is little information on the association with blood pressure when direct measures of blood long-chain n-3 PUFA levels, reflecting mainly intake of fish or fish oils, have been used, and the few results have been mixed. ${ }^{3,24,25}$ Therefore, the aim of this study was to investigate the association between serum long-chain n-3 PUFAs eicosapentaenoic acid (EPA), docosapentaenoic acid (DPA) and docosahexaenoic acid (DHA) on blood pressure in middle-aged and older men and women in Eastern Finland. We also investigated whether high hair methylmercury content, a marker of long-term

${ }^{1}$ University of Eastern Finland, Institute of Public Health and Clinical Nutrition, Kuopio, Finland and ${ }^{2}$ Department of Public Health, University of Eastern Africa, Baraton, Kenya Correspondence: Dr JK Virtanen, University of Eastern Finland, Kuopio Campus, Institute of Public Health and Clinical Nutrition, PO Box 1627, 70211-Kuopio, Finland. E-mail: jyrki.virtanen@uef.fi

Received 30 December 2011; revised and accepted 2 April 2012; published online 7 June 2012 
methylmercury exposure, is associated with blood pressure, which could partly explain the higher risk of CVD. ${ }^{19}$

\section{METHODS}

\section{Study population}

The KIHD Study is an ongoing population-based study designed to investigate risk factors for CVD and other chronic diseases in middle-aged and older men and women in Eastern Finland. ${ }^{26}$ The study protocol was approved by the Research Ethics Committee of the University of Kuopio. All subjects gave their written informed consent. The KIHD complies with the principles of the Declaration of Helsinki.

The baseline examinations of the KIHD Study were conducted between 1984 and 1989 to a random sample of men living in the city of Kuopio and neighboring rural communities. A total of 2682 men who were $42,48,54$ or 60 years old at baseline $(82.9 \%$ of those eligible) were recruited in two cohorts. The first cohort consisted of 1166 men who were 54 years old, enrolled between 1984 and 1986, and the second cohort included 1516 men who were 42, 48, 54 or 60 years old, enrolled between 1986 and 1989. During the years 1998-2001, all men from the second cohort were invited to the 11-year re-examinations of the study, and 854 men (85.6\%) participated. These examinations were also the baseline for 920 postmenopausal women $(78.4 \%$ of the 1173 eligible women) from the same area, aged 53-73 years. These 1774 men and women were used as the study population for the current study. For the present study we included 848 men and 909 women, for whom information on blood pressure, serum fatty acids and pubic hair mercury was available. In order to remove sources of possible bias, we further excluded participants with ischemic heart disease, stroke, diabetes or treatment for hypertension, which left 396 men and 372 women for the analyses.

\section{Blood pressure measurements}

All blood pressure measurements were performed by specially trained nurses. Resting blood pressure was measured between 0800 and $1000 \mathrm{~h}$ on the first examination day by one nurse with a random zero mercury sphygmomanometer with cuff size of $120 \times 350 \mathrm{~mm}$ or $150 \times 430 \mathrm{~mm}$. The measuring protocol included, after a supine rest of $5 \mathrm{~min}$, three measurements in supine, one in standing and two in sitting position with 5-min intervals. The mean of all six systolic pressure values was used in the present analyses as the systolic blood pressure and the mean of all six diastolic measurements as diastolic blood pressure. Pulse pressure was defined as the difference between the systolic and diastolic blood pressure.

\section{Serum fatty acids}

Serum esterified and nonesterified fatty acids were determined in one gas chromatographic run without preseparation as described. ${ }^{27}$ Fatty acids were chromatographed in an NB-351 capillary column (HNU-Nordion, Helsinki, Finland) by a Hewlett-Packard 5890 Series II gas chromatograph (HewlettPackard Company, Avondale, PA, USA) with a flame ionization detector. The coefficient of variation for repeated measurements of major esterified fatty acids was about $5 \%$. Because the relative degree of saturation of fatty acids varies among esterified fatty acid types (that is, cholesterol esters, phospholipids, and triglycerides), the esterified fatty acid concentrations were adjusted for serum low-density lipoprotein cholesterol, high-density lipoprotein cholesterol and triglyceride concentrations. The coefficient of variation for major nonesterified fatty acids was about $15 \%$. No adjustment was conducted for nonesterified fatty acids.

\section{Other measurements}

The subjects gave fasting blood samples between 0800 and $1000 \mathrm{~h}$ on the baseline examinations in 1998-2001. They were instructed to abstain from ingesting alcohol for 3 days and from smoking and eating for $12 \mathrm{~h}$ before giving sample. Detailed descriptions of the determination of blood glucose, ${ }^{28}$ assessment of medical history and medications, ${ }^{28}$ family history of diseases, ${ }^{28}$ smoking, ${ }^{28}$ and alcohol consumption ${ }^{28}$ have been published. Diabetes was defined as self-reported diabetes mellitus or fasting blood glucose of $6.7 \mathrm{mmoll}^{-1}$ or more. Education was assessed in years by using self-administrated questionnaire. Physical activity was assessed using the KIHD 12-Month Leisure-Time Physical Activity Questionnaire. ${ }^{29}$ Body mass index was computed as the ratio of weight in kilograms to the square of height in meters. Dietary intake of foods and nutrients was assessed at the time of blood sampling using 4-day food recording. ${ }^{30}$ Mercury in hair was determined by flow injection analysis-cold vapor atomic absorption spectrometry and amalgamation, as described. ${ }^{31}$

\section{Statistical analysis}

The relationships between baseline characteristics and serum EPA + DPA + DHA were explored by means and linear (for continuous variables) or logistic regression (for dichotomous variables). The mean values of systolic and diastolic blood pressure and pulse pressure in quartiles of serum EPA+ DPA + DHA and hair mercury were analyzed using analysis of covariance (ANCOVA). The linear relationship $(\beta)$ of serum fatty acids and hair mercury with systolic and diastolic blood pressure and pulse pressure were analyzed with multiple linear regression models, using log-transformed values. The Model 1 was adjusted for age, gender and examination year. The Model 2 included the Model 1 and hypertension in family, leisure-time physical activity, alcohol consumption, body mass index, years of education, employment status, 24-h urinary potassium and sodium excretion, and smoking. The cohort mean was used to replace missing values of covariates $(<2.9 \%$ of values). Few participants reported using fish oil supplements $(n<10)$. Tests of linear trend across quartiles of serum fatty acids or hair mercury were conducted by assigning the median values for each category of exposure variable and treating those as a single continuous variable. Statistical significance of the interactions on a multiplicative scale was assessed by likelihood ratio tests with a cross-product term. All $P$-values were 2-tailed $(\alpha=0.05)$. Data were analyzed with SPSS 14.0 for Windows (SPSS Inc, Chicago, IL, USA).

\section{RESULTS}

The mean serum concentrations were $1.63 \%$ (s.d. 0.91) for EPA, $0.77 \%$ (s.d. 0.16) for DPA and $2.73 \%$ (s.d. 0.90) for DHA (as a percentage of all serum fatty acids). Table 1 shows the characteristics of the study population. Those with a higher serum EPA + DPA + DHA concentration were more likely to have a higher income, education, hair mercury content, and urinary potassium excretion, and were less likely to smoke. They also had a higher intake of fish and vegetables (excluding potatoes) and a lower intake of energy and meat and meat products.

After adjusting for age, gender and examination year, a higher serum EPA + DPA + DHA concentration was statistically significantly associated with a lower systolic blood pressure and pulse pressure, but not with diastolic blood pressure (Model 1 in Table 2). The regression coefficients $(\beta)$ for the log-transformed serum EPA + DPA + DHA from the multiple linear regression models were $-5.01 \quad(95 \%$ confidence interval (CI) $-8.59--1.42$ ) for systolic blood pressure, $-0.50(95 \%$ CI $-2.43-1.43)$ for diastolic blood pressure and -4.51 (95\% CI $-7.02--2.00)$ for pulse pressure. Further multivariate adjustments slightly attenuated the associations (Model 2 in Table 2; systolic blood pressure: $\beta=-4.50,95 \%$ CI $-8.02--0.99$; diastolic blood pressure: $\beta=-0.45,95 \%$ CI $-2.32-1.42$; and pulse pressure: $\beta=-4.41,95 \%$ CI $-6.95--1.87)$. Further adjustments for serum 25-hydroxyvitamin D, use of aspirin or multivitamins, place of residence, total energy intake, milk and milk products, fruits and berries, vegetables and meat and meat products did not appreciably change the associations ( $<5 \%$ change). When we evaluated the EPA, DPA and DHA individually, we did not find significant differences between the fatty acids (Table 2).

The mean hair mercury concentration was $1.42 \mu \mathrm{gg}^{-1}$ (s.d. 1.54). We did not find any associations between hair mercury and blood pressure (Table 3). The multivariate-adjusted (Model 2) regression 
Table 1 Baseline characteristics of the KIHD Study population

Serum $E P A+D P A+D H A$ quartile (\% of serum fatty acids)

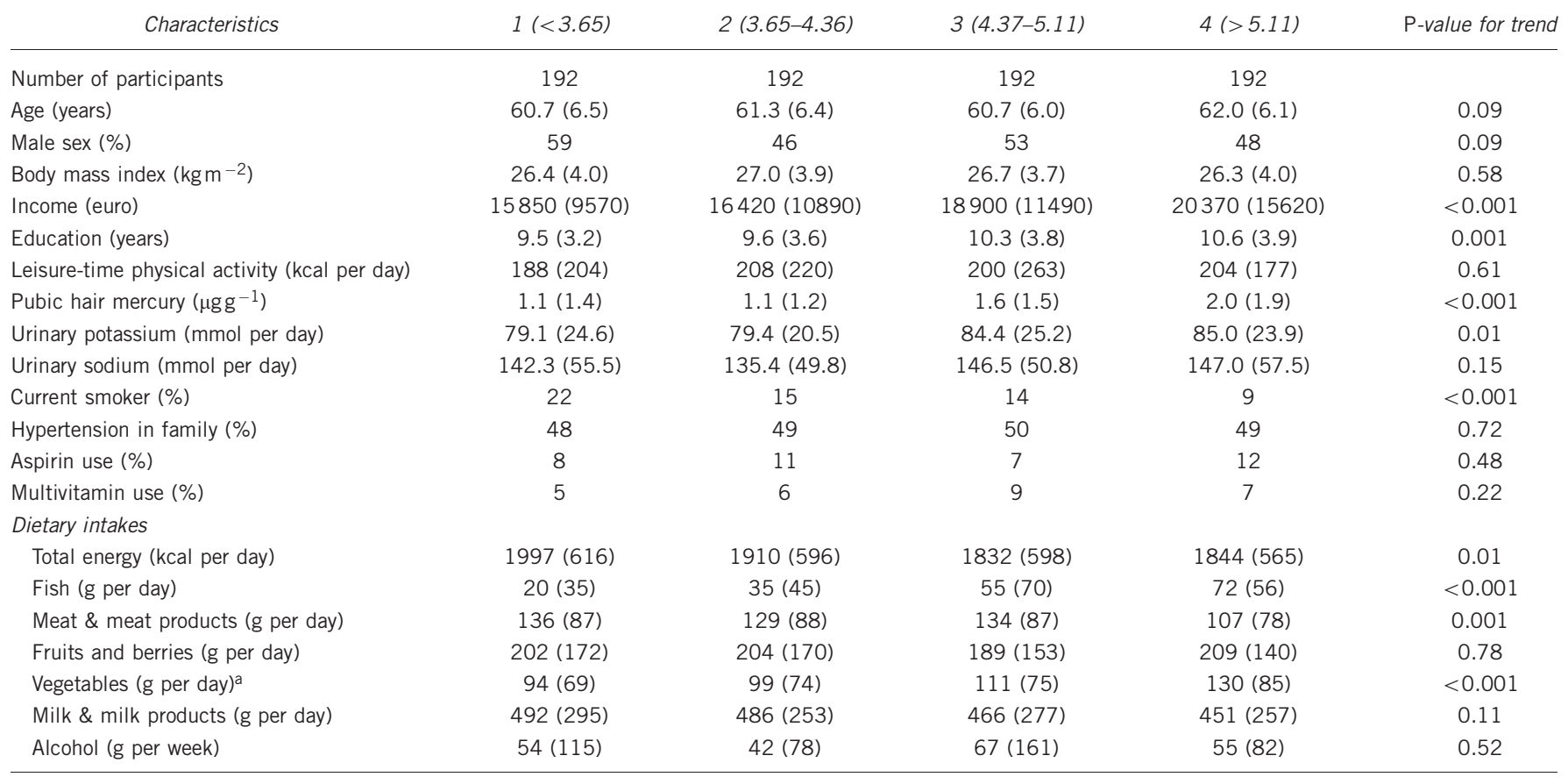

Abbreviations: CHD, coronary heart disease; DHA, docosahexaenoic acid; DPA, docosapentaenoic acid; EPA, eicosapentaenoic acid; KIHD, Kuopio Ischaemic Heart Disease. All values are mean (s.d.) or percentage.

${ }^{a}$ Excluding potatoes.

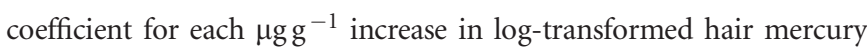
concentration was $-0.90(95 \%$ CI $-3.21-1.42)$ for systolic blood pressure, $-0.50(95 \%$ CI $-1.72-0.73)$ for diastolic blood pressure and $-0.40(95 \%$ CI $-2.08-1.28)$ for pulse pressure. Further adjustment for serum EPA+DPA+DHA did not change the associations (data not shown). High hair mercury content did not modify the associations between EPA + DPA + DHA and blood pressure, either $(P$ for interactions $>0.10)$

We did not find evidence for effect modification between serum $\mathrm{EPA}+\mathrm{DPA}+\mathrm{DHA}$ or hair mercury and blood pressure by age or gender $(P$ for interactions $>0.10)$.

\section{DISCUSSION}

The results from this cross-sectional cohort study suggest that a higher serum concentration of total long-chain n-3 PUFA, mainly reflecting fatty fish or fish oil consumption, is associated with a modestly lower systolic blood pressure and pulse pressure, whereas exposure to methylmercury has no association with the risk.

Although the mean difference in systolic blood pressure and pulse pressure between the extreme quartiles was only $4 \mathrm{~mm} \mathrm{Hg}$ in this study, this may have clinical relevance. It has been estimated that a $2 \mathrm{~mm} \mathrm{Hg}$ reduction in systolic blood pressure can reduce coronary artery disease mortality by $4 \% .{ }^{32}$ Because use of fish oil supplements in this study population is very low ( $<10$ subjects), the observed blood levels reflect regular fish consumption.

In meta-analyses of RCTs, fish oil supplementation has been shown to lower both systolic and diastolic blood pressure, but the effect has been stronger in hypertensive subjects than in normotensives. ${ }^{6-8}$ In the most recent meta-analysis, in normotensive subjects fish oil supplementation lowered systolic and diastolic blood pressure, on average, by $1.0 \mathrm{~mm} \mathrm{Hg}$ and $1.2 \mathrm{~mm} \mathrm{Hg}$, respectively. ${ }^{8}$ In hypertensive subjects, the average reductions were $4.0 \mathrm{~mm} \mathrm{Hg}$ and $2.5 \mathrm{~mm} \mathrm{Hg}$, respectively. Older meta-analyses of fish oil supplementation found similar reductions in blood pressure. ${ }^{6,7}$ On the other hand, a recent cross-over trial of low-dose DHA supplementation found an average reduction of $3.3 \mathrm{~mm} \mathrm{Hg}$ in diastolic blood pressure in normotensive subjects. ${ }^{33}$ The duration of the RCTs have usually been from a few weeks to a maximum of 1 year, and the doses of fish oil have generally been large (usually $\geqslant 3 \mathrm{~g} \mathrm{~d}^{-1}$ ), ${ }^{8}$ which is difficult to obtain from diet alone. However, a modest inverse association between dietary intakes of fish or long-chain n-3 PUFA or blood levels of these fatty acids and blood pressure has been observed in observational studies in general populations, ${ }^{2-5,25}$ and also in healthy, normotensive participants, ${ }^{5}$ although not all studies have found an association. ${ }^{24}$

In comparing EPA and DHA, we did not find significant differences with regard to their association with blood pressure. We have previously observed a stronger association with DHA than with EPA in the prevention of atrial fibrillation in men in this study population, ${ }^{34}$ and a RCT found that DHA was more effective than EPA in lowering blood pressure. ${ }^{35}$ However, another RCT with similar design did not find an effect on blood pressure with either EPA or DHA. ${ }^{36}$ Thus, our results suggest that higher serum levels of both EPA and DHA are associated with lower blood pressure.

Besides being a source of long-chain n-3 PUFA, fish can also contain environmental pollutants, such as methylmercury. We have previously shown that higher hair mercury content is associated with increased risk of CVD in men in this study population. ${ }^{19,31}$ Other studies have found a positive association between mercury exposure and blood pressure not only in populations with high mercury exposure, ${ }^{20-23}$ but also among non-fish consumers. ${ }^{37}$ However, our 
Table 2 Blood pressure in quartiles of serum long-chain n-3 PUFA

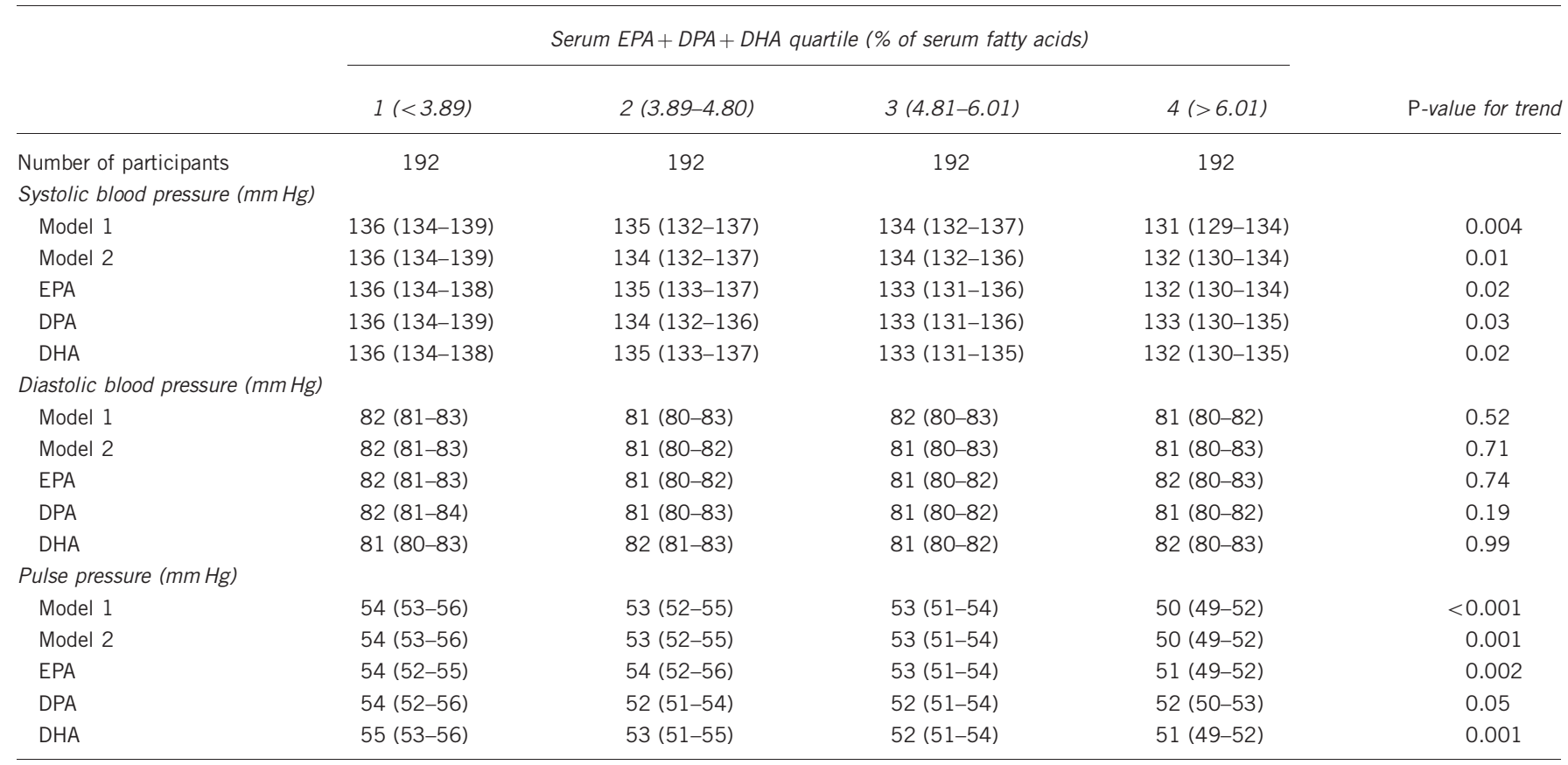

Abbreviations: $\mathrm{Cl}$, confidence interval; EPA, eicosapentaenoic acid; DPA, docosapentaenoic acid; DHA, docosahexaenoic acid; PUFA, polyunsaturated fatty acids. All values are means $(95 \% \mathrm{Cl})$, obtained with analysis of covariance.

Model 1: adjusted for age, gender and examination year.

Model 2: adjusted for model 1 and hypertension in family, leisure-time physical activity, alcohol consumption, body mass index, education, employment status, 24-h urinary potassium and sodium excretion, and smoking.

Table 3 Blood pressure in quartiles of hair mercury

\begin{tabular}{|c|c|c|c|c|c|}
\hline & \multicolumn{4}{|c|}{ Hair mercury quartile, $\mu g g^{-1}$} & \multirow[b]{2}{*}{ P-value for trend } \\
\hline & $1(<0.42)$ & $2(0.42-0.88)$ & $3(0.89-1.78)$ & $4(>1.78)$ & \\
\hline \multicolumn{6}{|c|}{ Systolic blood pressure $(\mathrm{mm} \mathrm{Hg})$} \\
\hline Model 1 & $135(133-138)$ & $133(130-135)$ & $133(130-135)$ & $136(133-138)$ & 0.37 \\
\hline Model 2 & $135(133-138)$ & $134(132-136)$ & $133(131-135)$ & $134(132-137)$ & 0.87 \\
\hline Model 2 & $82(81-83)$ & $81(80-83)$ & $81(80-82)$ & $81(80-83)$ & 0.52 \\
\hline \multicolumn{6}{|l|}{ Pulse pressure $(\mathrm{mm} \mathrm{Hg})$} \\
\hline Model 1 & $53(52-55)$ & $52(50-53)$ & $52(50-54)$ & $54(52-55)$ & 0.35 \\
\hline Model 2 & $53(52-55)$ & $52(51-54)$ & $52(51-54)$ & $53(51-55)$ & 0.81 \\
\hline
\end{tabular}

Abbreviation: $\mathrm{Cl}$, confidence interval.

All values are means $(95 \% \mathrm{CI})$, obtained from the analysis of covariance.

Model 1: adjusted for age, gender and examination year.

Model 2: adjusted for model 1 and hypertension in family, leisure-time physical activity, alcohol consumption, body mass index, education, employment status, 24-h urinary potassium and sodium excretion, and smoking.

results indicate that a moderate mercury exposure is not associated with blood pressure, and also the overall evidence regarding the association between mercury exposure and blood pressure is considered weak. ${ }^{38}$ Thus, other mechanisms, such as lipid peroxidation, ${ }^{31}$ may explain the increased risk of CVD in this study population.

Strength of the present study is the use of serum long-chain n-3 PUFA measurements instead of dietary intakes. Few prior studies have used circulating long-chain n-3 PUFA concentration as an exposure, although circulating n-3 PUFA is an objective marker of exposure to these fatty acids and thus reduces bias by misclassification that often attenuates the associations in studies using dietary intakes. Serum long-chain n-3 PUFA concentration is a good marker for long-chain n-3 PUFA intake from fish; it correlates well with dietary intakes of fish or fish oil, ${ }^{39}$ and reflects dietary intakes during the preceding weeks. ${ }^{40}$ Other strengths of the study are its population-based recruitment, high participation rate and extensive examinations of potential risk factors. The cross-sectional design is a potential weakness.

In conclusion, in older men and women, higher serum long-chain n-3 PUFA levels, mainly reflecting fish intake in this study population, were associated with a modestly lower blood pressure. On the other hand, mercury, an environmental pollutant in fish, was not associated 
with blood pressure. This suggests that fish consumption may help in blood pressure regulation.

\section{CONFLICT OF INTEREST}

The authors declare no conflict of interest.

\section{ACKNOWLEDGEMENTS}

This study was supported by the Academy of Finland grant no.121206 to JKV.

1 Mozaffarian D, Wu JH. Omega-3 fatty acids and cardiovascular disease: Effects on risk factors, molecular pathways, and clinical events. J Am Coll Cardiol 2011; 58: 2047-2067.

2 Pauletto P, Puato M, Caroli MG, Casiglia E, Munhambo AE, Cazzolato G, Bittolo BG, Angeli MT, Galli C, Pessina AC. Blood pressure and atherogenic lipoprotein profiles of fish-diet and vegetarian villagers in Tanzania: The Lugalawa study. Lancet 1996; 348: 784-788.

3 Dallongeville J, Yarnell J, Ducimetiere P, Arveiler D, Ferrieres J, Montaye M, Luc G, Evans A, Bingham A, Hass B, Ruidavets JB, Amouyel P. Fish consumption is associated with lower heart rates. Circulation 2003; 108: 820-825.

4 Mozaffarian D, Gottdiener JS, Siscovick DS. Intake of tuna or other broiled or baked fish versus fried fish and cardiac structure, function, and hemodynamics. Am J Cardiol 2006; 97: 216-222.

5 Ueshima H, Stamler J, Elliott P, Chan Q, Brown IJ, Carnethon MR, Daviglus ML, He K, Moag-Stahlberg A, Rodriguez BL, Steffen LM, Van Horn L, Yarnell J, Zhou B. INTERMAP Research Group. Food omega-3 fatty acid intake of individuals (total, linolenic acid, long-chain) and their blood pressure: INTERMAP study. Hypertension 2007; 50: 313-319.

6 Morris MC, Sacks F, Rosner B. Does fish oil lower blood pressure? A meta-analysis of controlled trials. Circulation 1993; 88: 523-533.

7 Appel LJ, Miller 3rd ER, Seidler AJ, Whelton PK. Does supplementation of diet with 'fish oil' reduce blood pressure? A meta-analysis of controlled clinical trials. Arch Intern Med 1993; 153: 1429-1438.

8 Geleijnse JM, Giltay EJ, Grobbee DE, Donders AR, Kok FJ. Blood pressure response to fish oil supplementation: Metaregression analysis of randomized trials. J Hypertens 2002; 20: 1493-1499.

9 Erkkila AT, Schwab US, de Mello VD, Lappalainen T, Mussalo H, Lehto S, Kemi V Lamberg-Allardt C, Uusitupa MI. Effects of fatty and lean fish intake on blood pressure in subjects with coronary heart disease using multiple medications. Eur J Nutr 2008, 47: $319-328$

10 Lara JJ, Economou M, Wallace AM, Rumley A, Lowe G, Slater C, Caslake M, Sattar N, Lean ME. Benefits of salmon eating on traditional and novel vascular risk factors in young, non-obese healthy subjects. Atherosclerosis 2007; 193: 213-221.

11 Ness AR, Whitley E, Burr ML, Elwood PC, Smith GD, Ebrahim S. The long-term effect of advice to eat more fish on blood pressure in men with coronary disease: Results from the Diet and Reinfarction Trial. J Hum Hypertens 1999; 13: 729-733.

12 Vandongen R, Mori TA, Burke V, Beilin LJ, Morris J, Ritchie J. Effects on blood pressure of omega 3 fats in subjects at increased risk of cardiovascular disease. Hypertension 1993; 22: 371-379.

13 Cobiac L, Clifton PM, Abbey M, Belling GB, Lipid Nestel PJ.. lipoprotein, and hemostatic effects of fish vs fish-oil n-3 fatty acids in mildly hyperlipidemic males. Am J Clin Nutr 1991; 53: 1210-1216.

14 Yin K, Chu ZM, Beilin LJ. Blood pressure and vascular reactivity changes in spontaneously hypertensive rats fed fish oil. Br J Pharmacol 1991; 102: 991-997.

15 Mori TA, Watts GF, Burke V, Hilme E, Puddey IB, Beilin LJ. Differential effects of eicosapentaenoic acid and docosahexaenoic acid on vascular reactivity of the forearm microcirculation in hyperlipidemic, overweight men. Circulation 2000; 102 1264-1269.

16 Nestel P, Shige H, Pomeroy S, Cehun M, Abbey M, Raederstorff D. The n-3 fatty acids eicosapentaenoic acid and docosahexaenoic acid increase systemic arterial compliance in humans. Am J Clin Nutr 2002; 76: 326-330.

17 Mozaffarian D, Geelen A, Brouwer IA, Geleijnse JM, Zock PL, Katan MB. Effect of fish oil on heart rate in humans: A meta-analysis of randomized controlled trials. Circulation 2005; 112: 1945-1952.
18 Engler MM, Engler MB, Pierson DM, Molteni LB, Molteni A. Effects of docosahex aenoic acid on vascular pathology and reactivity in hypertension. Exp Biol Med 2003 228: 299-307.

19 Virtanen JK, Voutilainen S, Rissanen TH, Mursu J, Tuomainen TP, Korhonen MJ, Valkonen VP, Seppanen K, Laukkanen JA, Salonen JT. Mercury, fish oils, and risk of acute coronary events and cardiovascular disease, coronary heart disease, and allcause mortality in men in eastern Finland. Arterioscler Thromb Vasc Biol 2005; 25: 228-233.

20 Fillion M, Mergler D, Sousa Passos CJ, Larribe F, Lemire M, Guimaraes JR. A preliminary study of mercury exposure and blood pressure in the Brazilian Amazon. Environ Health 2006; 5: 29

21 Pedersen EB, Jorgensen ME, Pedersen MB, Siggaard C, Sorensen TB, Mulvad G, Hansen JC, Asmund G, Skjoldborg H. Relationship between mercury in blood and 24-h ambulatory blood pressure in Greenlanders and Danes. Am J Hypertens 2005; 18: 612-618.

22 Valera B, Dewailly E, Poirier P. Environmental mercury exposure and blood pressure among Nunavik inuit adults. Hypertension 2009; 54: 981-986.

23 Choi AL, Weihe P, Budtz-Jorgensen E, Jorgensen PJ, Salonen JT, Tuomainen TP, Murata K, Nielsen HP, Petersen MS, Askham J, Grandjean P. Methylmercury exposure and adverse cardiovascular effects in Faroese whaling men. Environ Health Perspect 2009; 117: 367-372.

24 Simon JA, Fong J, Bernert Jr. JT. Serum fatty acids and blood pressure. Hypertension 1996; 27: 303-307.

25 Ebbesson SO, Risica PM, Ebbesson LO, Kennish JM, Tejero ME. Omega-3 fatty acids improve glucose tolerance and components of the metabolic syndrome in Alaskan Eskimos: The Alaska Siberia project. Int J Circumpolar Health 2005; 64: 396-408.

26 Salonen JT. Is there a continuing need for longitudinal epidemiologic research? The Kuopio Ischemic Heart Disease Risk Factor Study. Ann Clin Res 1988; 20: 46-50.

27 Laaksonen DE, Lakka TA, Lakka HM, Nyyssonen K, Rissanen T, Niskanen LK, Salonen JT. Serum fatty acid composition predicts development of impaired fasting glycaemia and diabetes in middle-aged men. Diabet Med 2002; 19: 456-464.

28 Salonen JT, Nyyssonen K, Korpela H, Tuomilehto J, Seppanen R, Salonen R. High stored iron levels are associated with excess risk of myocardial infarction in eastern Finnish men. Circulation 1992; 86: 803-811.

29 Lakka TA, Venäläinen JM, Rauramaa R, Salonen R, Tuomilehto J, Salonen JT. Relation of leisure-time physical activity and cardiorespiratory fitness to the risk of acute myocardial infarction. N Engl J Med 1994; 330: 1549-1554.

30 Voutilainen S, Rissanen TH, Virtanen J, Lakka TA, Salonen JT. Low dietary folate intake is associated with an excess incidence of acute coronary events: The Kuopio Ischemic Heart Disease Risk Factor Study. Circulation 2001; 103: 2674-2680.

31 Salonen JT, Seppanen K, Nyyssonen K, Korpela H, Kauhanen J, Kantola M, Tuomilehto J, Esterbauer H, Tatzber F, Salonen R. Intake of mercury from fish, lipid peroxidation, and the risk of myocardial infarction and coronary, cardiovascular, and any death in eastern Finnish men. Circulation 1995; 91: 645-655.

32 Stamler J, Rose G, Stamler R, Elliott P, Dyer A, Marmot M. INTERSALT study findings. Public health and medical care implications. Hypertension 1989; 14: 570-577.

33 Theobald HE, Goodall AH, Sattar N, Talbot DC, Chowienczyk PJ, Sanders TA. Low-dose docosahexaenoic acid lowers diastolic blood pressure in middle-aged men and women. J Nutr 2007; 137: 973-978.

34 Virtanen JK, Mursu J, Voutilainen S, Tuomainen TP. Serum long-chain n-3 polyunsaturated fatty acids and risk of hospital diagnosis of atrial fibrillation in men. Circulation 2009; 120: 2315-2321.

35 Mori TA, Bao DQ, Burke V, Puddey IB, Beilin LJ. Docosahexaenoic acid but not eicosapentaenoic acid lowers ambulatory blood pressure and heart rate in humans. Hypertension 1999; 34: 253-260.

36 Woodman RJ, Mori TA, Burke V, Puddey IB, Watts GF, Beilin LJ. Effects of purified eicosapentaenoic and docosahexaenoic acids on glycemic control, blood pressure, and serum lipids in type 2 diabetic patients with treated hypertension. Am J Clin Nut 2002; 76: 1007-1015.

37 Vupputuri S, Longnecker MP, Daniels JL, Guo X, Sandler DP. Blood mercury level and blood pressure among US women: results from the National Health and Nutrition Examination Survey 1999-2000. Environ Res 2005; 97: 195-200.

38 Roman HA, Walsh TL, Coull BA, Dewailly E, Guallar E, Hattis D, Marien K, Schwartz J, Stern AH, Virtanen JK, Rice G. Evaluation of the cardiovascular effects of methylmercury exposures: current evidence supports development of a dose-response function for regulatory benefits analysis. Environ Health Perspect 2011; 119: 607-614.

39 Hunter D. Biochemical indicators of dietary intake. In: Willett W (ed.), Nutritional Epidemiology. Oxford University Press, New York, NY, 1998, p174.

40 Nikkari T, Luukkainen P, Pietinen P, Puska P. Fatty acid composition of serum lipid fractions in relation to gender and quality of dietary fat. Ann Med 1995; 27: 491-498. 